\title{
1 Effects of soil preservation for biodiversity monitoring using environmental DNA
}

4 Alessia Guerrieri" * , Aurélie Bonin¹, Tamara Münkemüller², Ludovic Gielly², Wilfried Thuiller², Gentile

5 Francesco Ficetola, 2

6

7 Affiliations:

$8{ }^{1}$ Department of Environmental Science and Policy, Università degli Studi di Milano, Via Celoria 26, 20133

9 Milano, Italy

10 '2Univ. Grenoble Alpes, CNRS, Univ. Savoie Mont Blanc, LECA, Laboratoire d'Ecologie Alpine, F-38000,

11 Grenoble, France

12

*Correspondence author. Email: alessia.guerrieri@unimi.it 


\section{ABSTRACT}

Environmental DNA metabarcoding is becoming a key tool for biodiversity monitoring over large geographical or taxonomic scales and for elusive taxa like soil organisms. Increasing sample sizes and interest in remote or extreme areas often require the preservation of soil samples and thus deviations from optimal standardized protocols. However, we still ignore the impact of different methods of soil sample preservation on the results of metabarcoding studies and there is no guideline for best practices so far. Here, we assessed the impact of four methods of soil sample preservation that can be conveniently used also in metabarcoding studies targeting remote or difficult to access areas. The tested methods include: preservation at room temperature for $6 \mathrm{~h}$, preservation at $4^{\circ} \mathrm{C}$ for three days, desiccation immediately after sampling and preservation for 21 days, and desiccation after $6 \mathrm{~h}$ at room temperature and preservation for 21 days. For each preservation method, we benchmarked resulting estimates of taxon diversity and community composition of three different taxonomic groups (bacteria, fungi and eukaryotes) in three different habitats (forest, river bank and grassland) against results obtained under ideal conditions (i.e. extraction of eDNA right after sampling). Overall, the different preservation methods only marginally impaired results and only under certain conditions. When rare taxa were considered, we detected small but significant changes in MOTU richness of bacteria, fungi and eukaryotes across treatments, but MOTUs richness was similar across preservation methods if rare taxa were not considered. All the approaches were able to identify differences in community structure among habitats, and the communities retrieved using the different preservation conditions were extremely similar. We propose guidelines on the selection of the optimal soil sample preservation conditions for metabarcoding studies, depending on the practical constraints, costs and ultimate research goals.

KEYWORDS: eDNA metabarcoding, sample storage, MOTU richness, $\alpha$ and $\beta$ diversity, microbial communities, eukaryotes 


\section{INTRODUCTION}

Environmental DNA (hereafter referred to as "eDNA") can be defined as the mixture of complex, sometimes degraded, DNA that microorganisms (e.g. bacteria and fungi) or macroorganisms (e.g. animals, plants) leave behind in their environment (i.e. soil, water, sediments, etc.). By studying short, taxonomically-informative DNA fragments obtained from eDNA samples, it is possible to identify the associated taxa and therefore to survey biodiversity. Coined as "eDNA metabarcoding", this approach has revolutionized several branches of ecology and environmental sciences during the last decade, by providing relatively quick and non-invasive assessments of present or past biodiversity of animals, plants and microorganisms (Taberlet, Bonin, Zinger, \& Coissac, 2018). Metabarcoding is particularly valuable for monitoring biodiversity over large geographical or taxonomic scales (De Vargas et al., 2015; Delgado-Baquerizo et al., 2018; Zinger et al., 2019b).

Furthermore, it gives access to biodiversity components that are elusive to conventional survey methods. For instance, it allows the rapid assessment of microbial soil biodiversity, which is extremely complex, timeconsuming and imperfect when using direct observations, culturing techniques or microscopy (Giovannoni, Britschgi, Moyer, \& Field, 1990; Ward, Weller, \& Bateson, 1990).

Metabarcoding relies on a succession of several steps: 1) sampling; 2) preservation of the collected material until lab processing; 3) DNA extraction; 4) PCR amplification of a selected genomic region; 5) highthroughput sequencing of amplicons; and 6) analysis of sequences using bioinformatics and statistical tools (Zinger, Bonin, et al., 2019). Each step is critical to obtain robust taxonomic inventories and diversity estimates, and an increasing number of studies has assessed how methodological choices across the different steps could influence the conclusions of a study (Calderón-Sanou, Münkemüller, Boyer, Zinger, \& Thuiller, 2020; Cantera et al., 2019; Chen \& Ficetola, 2020; Nichols et al., 2018; Taberlet et al., 2018). Despite this growing body of literature, So far little attention has been devoted to the effect of different preservation conditions of the collected environmental material before lab processing (i.e. step 2). We thus know little about the optimal storage conditions of the collected material, and how long samples can be stored to limit biases in taxonomic inventories. 
Some recent studies have analyzed the preservation of sampling material from obtained from water (see e.g. Kumar, Eble, \& Gaither, 2020; Majaneva et al., 2018). Conversely, methodological analyses on the effects of sample preservation are largely scarce for soil, perhaps because the majority of metabarcoding studies have so far been performed in temperate areas where access to lab facilities is often easy (Hoffmann, Schubert, \& Calvignac-Spencer, 2016; Huerlimann et al., 2020). In such cases, sample preservation is sometimes not necessary at all, or at least not over long periods of time. However, one great promise of metabarcoding is its potential for providing biodiversity data for remote areas, where biodiversity monitoring is essential but difficult. When sampling in remote or inaccessible areas (e.g. tropical and arctic areas; mountain chains), samples are rarely collected nearby lab facilities and an immediate in situ DNA extraction is generally not possible due to logistic constraints (but see Zinger, Taberlet, et al., 2019b for a notable exception). More generally, with the ever-increasing number of samples analyzed during a typical metabarcoding study, sample preservation is more and more indispensable, and the time lag between sample collection and subsequent molecular processing makes it particularly relevant to understand the impact of sample preservation, and to identify preservation strategies that do not bias the conclusions of studies.

In an ideal metabarcoding study, communities recovered from preserved samples should match those retrieved if samples had been processed immediately after sampling. However, inappropriate preservation conditions can cause both DNA degradation and the proliferation of certain taxonomic groups with respect to others, before DNA extraction (Cardona et al., 2012; Orchard, Standish, Nicol, Dickie, \& Ryan, 2017). This can in turn affect taxa detection and also the relative contributions of different taxonomic groups to the estimated biodiversity. A recent review suggested that the majority of eDNA metabarcoding studies does not provide accurate information about sample treatment before processing (Dickie et al., 2018). Almost half of the studies do not report how samples were stored and conserved, and $30 \%$ of them store samples at $0-4^{\circ} \mathrm{C}$, and thus at a temperature where many bacteria and fungi continue to be active and potentially affecting the whole sample. About $15 \%$ of the studies stored samples in a range of $5-35^{\circ} \mathrm{C}$, which 
can be a poor practice when no preservatives are added (Dickie et al., 2018), and only $10 \%$ stored them below $0^{\circ} \mathrm{C}$ (Dickie et al., 2018).

So far, the consequences of preservation practices and the resulting deviations from immediate processing and analyses have rarely been studied quantitatively. Some studies suggested that liquid-based preservatives have limited usefulness for soil eDNA (Tatangelo et al. 2014). Yet, Lauber, Zhou, Gordon, Knight, \& Fierer (2010) tested the effect of storing samples from soil, human gut and skin at different temperatures and did not detect any significant effect on bacterial communities, while Orchard et al. (2017) found that storage time and temperature can affect colonization by arbuscular mycorrhizal fungi, with subsequent impacts on the reconstruction of communities. Differences between these studies may be due to their different protocols. However, they also focused on different taxonomic groups, which may react differently to storage period and temperature. Consequently, these studies are difficult to compare, highlighting the importance of formal assessments of preservation methods. Desiccation is a further approach that can efficiently conserve high-quality DNA for genomic studies (e.g. Chase \& Hills, 1991). Although not widely used for metabarcoding samples, desiccation through silica gel has good potential for soil sample preservation, as it allows removing $>25 \%$ of its weight in water in a few hours (P. Taberlet, pers. communication), is cost-effective, easy to transport, and is not an issue for aircraft transportation (no flammable or dangerous preservatives). A clear understanding of the effect of different preservation methods, especially across various groups of taxa, is thus pivotal for a robust application of eDNA metabarcoding to biodiversity monitoring in general, and that of remote areas in particular. Here, using eDNA metabarcoding of different taxonomic groups in soil systems, we tested: (i) how preservation methods influence overall richness estimates and what the role of rarely observed taxa is; (ii) how preservation methods influence identified community structure and its turnover between different habitats; and (iii) what the best practices are under limited laboratory access. More specifically, we first selected three soil preservation methods (room temperature, $4^{\circ} \mathrm{C}$, desiccation in silica gel) because they are commonly used in the literature (room temperature and $4^{\circ} \mathrm{C}$ ) or because they are easy to implement in the field (desiccation and room temperature). Then, we assessed the impact of these preservation methods 
114 applied to different durations in order to mimic logistic constraints (see Supplementary Material, Appendix

115 A for details on experimental design), and compared the communities obtained with those observed in

116 ideal conditions, i.e. when eDNA is extracted immediately after sampling (within less than one hour). We

117 examined bacterial, fungal and eukaryotic communities to cover a broad taxonomic range, since different

118 taxa can be differentially affected by sample preservation conditions (Cardona et al., 2012; Orchard et al.,

119 2017).

120 
Soil preservation and experimental treatments

In April 2019, we collected soil samples from three habitats: a grassland ( $\mathrm{N} 45.194^{\circ} \mathrm{E} 5.776^{\circ}$ ), a broadleaved forest ( $\left.\mathrm{N} 45.196^{\circ} \mathrm{E} 5.774^{\circ}\right)$, and a vegetated river bank ( $\left.\mathrm{N} 45.195^{\circ} \mathrm{E} 5.780^{\circ}\right)$. The study design was optimized to allow DNA extraction immediately after sampling, which hampered using distant study sites. All sites were within $400 \mathrm{~m}$ from the Laboratoire d'Écologie Alpine (LECA) in Grenoble, France. We choose habitats with some differentiation to allow different communities but not too extreme and relatively close together so that we expect some overlap between communities. This mimics what is commonly done in the field when gradients are sampled. We established five plots within each habitat; the minimum distance between nearby plots was about $20 \mathrm{~m}$. Within each plot, we collected four soil samples (with a minimum distance of one meter) at a depth of 0-20 cm and then pooled them together, for a total of five pooled samples per habitat (approx. $200 \mathrm{~g}$ each pooled sample). Soil litter was not included in the samples. Pooled samples (15 in total) were homogenized; subsequently, from each of them we took five subsamples of $15 \mathrm{~g}$ of soil (total: 75 subsamples; Fig. 1).

The five soil subsamples of each pooled sample were subjected to five different treatments: 1) eDNA was extracted immediately after sampling (within $1 \mathrm{~h}$; treatment hereafter referred to as "control"); 2 ) samples were preserved at room temperature $\left(21-23^{\circ} \mathrm{C}\right)$ and eDNA was extracted $6 \mathrm{~h}$ after sampling; 3 ) samples were inserted in sterile $50-\mathrm{mL}$ falcon tubes and preserved at $4^{\circ} \mathrm{C}$. eDNA was extracted three days after sampling; 4) samples were inserted in hermetic, sterile boxes with $20 \mathrm{~g}$ of silica gel immediately after sampling, then stored at room temperature, and eDNA was extracted 21 days after sampling; 5) samples were inserted in hermetic, sterile boxes with $20 \mathrm{~g}$ of silica gel $6 \mathrm{~h}$ after sampling, then stored at room temperature, and eDNA was extracted 21 days after sampling.

We did not test full-factorial combinations of different preservation periods and conditions, which was not feasible in terms of time and costs We did not consider freezing or storage in liquid nitrogen, which is unrealistic when dealing with large numbers and / or volumes of samples, as is the case for large-scale metabarcoding studies. Furthermore, freezing is generally impossible in remote areas (Dickie et al., 2018), 
where maintaining a cold chain cannot be ensured given the logistical challenges and is often replaced by preservation in a cool box $\left(4^{\circ} \mathrm{C}\right)$. Previous studies showed that preservative solutions have limited usefulness for soil or sediment samples (Rissanen et al., 2010; Tatangelo et al., 2014).

150

151

152

\section{Molecular analyses}

For all sample treatments, eDNA extraction was performed in a dedicated room using the NucleoSpin ${ }^{\circledR}$ Soil Mini Kit (Macherey-Nagel, Germany), after a preliminary step where $15 \mathrm{~g}$ of soil were mixed with $20 \mathrm{ml}$ of phosphate buffer for 15 minutes as described in (Taberlet, Coissac, Pompanon, Brochmann, \& Willerslev, 2012); and with a final elution in $150 \mu \mathrm{l}$. We also included one extraction negative control per treatment. Environmental DNA of bacteria, fungi and eukaryotes was amplified using primers designed for markers Bact02 (Taberlet et al., 2018), Fung02 (Epp et al., 2012; Taberlet et al., 2018) and Euka02 (Guardiola et al., 2015), respectively. Bact02 and Fung02 amplify fragments of about 220-250 bp, while Euka02 generally amplifies fragments $<150 \mathrm{bp}$. The three markers are well suited for metabarcoding analyses, as all have a very low number of mismatches in the priming region across target organisms, and the relatively short length of amplified fragments allows their use with potentially degraded DNA (Taberlet et al., 2018). To allow bioinformatic discrimination of PCR replicates after sequencing, eight-nucleotide long tags were added on the $5^{\prime}$ end of both forward and reverse primers, so that each PCR replicate was represented by a unique combination of forward and reverse tags. Tags had at least five nucleotide differences among them (Coissac, 2012). Samples were randomized on a 96-well plate, along with the five extraction controls, eight bioinformatic blanks, six PCR negative controls and two PCR positive controls. PCR positive controls were included to check for potential cross-contaminations and to monitor amplification and sequencing performances. The positive control was a 1:10 dilution of the ZymoBIOMICS ${ }^{\text {TM Microbial }}$ Community DNA Standard II (Zymo Research, USA) constituted of genomic DNA of eight bacterial and two fungal strains (i.e., Pseudomonas aeruginosa, Escherichia coli, Salmonella enterica, Lactobacillus fermentum, Enterococcus faecalis, Staphylococcus aureus, Listeria monocytogenes, Bacillus subtilis, Saccharomyces cerevisiae, Cryptococcus neoformans) at known concentrations. 
In order to avoid over-amplification of template DNA and to limit chimera formation, we determined the optimal number of amplification cycles and DNA extract dilution using qPCR. The qPCR assay was conducted on 48 randomly selected samples, using $1 \mu$ l of 1:1000 diluted SYBR ${ }^{\circledast}$ Green I nucleic acid gel stain (Invitrogen ${ }^{\mathrm{TM}}$, USA), with a real-time PCR thermal cycler set to standard mode. qPCR was performed for both 1:10 diluted and undiluted template eDNA.

For Bact02 and Fung02, PCR reactions were performed on 1:10 diluted template DNA, using 32 and 44 cycles respectively. For Euka02, we performed 34 cycles on undiluted DNA. All PCR reactions consisted of $10 \mu$ l of AmpliTaq Gold 360 Master Mix 2X (Applied Biosystems ${ }^{\mathrm{TM}}$, Foster City, CA, USA), $2 \mu$ l of primers mix at initial concentration of $5 \mu \mathrm{M}$ of each primer, $0.16 \mu \mathrm{l}$ of Bovine Serum Albumin (corresponding to $3.2 \mu \mathrm{g}$; Roche Diagnostic, Basel, Switzerland) and $2 \mu$ of DNA extract, for a final volume of $20 \mu \mathrm{l}$. The PCR profiles had an initial step of $10 \mathrm{~min}$ at $95^{\circ} \mathrm{C}$, followed by several cycles of a $30 \mathrm{~s}$ denaturation at $94^{\circ} \mathrm{C}$, a $30 \mathrm{~s}$ annealing at $53^{\circ} \mathrm{C}$ (Bact02), $56^{\circ} \mathrm{C}$ (Fung02) or $45^{\circ} \mathrm{C}$ (Euka02), and a $90 \mathrm{~s}$ elongation for Bact02 and Fung02, or a 60 s elongation for Euka02 at $72^{\circ} \mathrm{C}$, followed by a final elongation at $72^{\circ} \mathrm{C}$ for 7 minutes. The amplification was performed in 384-well plates, with four replicates for each sample. After amplification, PCR products of the same marker were pooled together in equal volumes and a 5 - $\mu$ l aliquot of the pooled amplicons was visualized by high-resolution capillary electrophoresis (QIAxcel Advanced System, QIAGEN, GERMANY) to verify the expected fragments length and to monitor primer dimers. Pooled amplicons were purified using the MinElute PCR Purification Kit (QIAGEN, GERMANY) following the manufacturer's protocol. Six subsamples of the pool of amplicons were purified separately for each marker, and then combined again before being sent for library preparation and sequencing to Fasteris (SA, Geneva, Switzerland). One library was prepared per marker using the MetaFast protocol (Taberlet et al., 2018) and then sequenced using the MiSeq (Fung02 and Bact02) or HiSeq 2500 (Euka02) platforms (Illumina, San Diego, CA, USA) with a paired-end approach ( $2 \times 250$ bp for Fung02 and Bact02, and $2 \times 150$ bp for Euka02).

\section{Bioinformatic treatment}


The bioinformatic treatment of sequence data was performed using the OBITools software suite (Boyer et al., 2016). First, forward and reverse reads were assembled using the illuminapairedend program, keeping only sequences with an alignment score higher than 40 . Aligned sequences were assigned to the corresponding PCR replicate using the program ngsfilter, by allowing two and zero mismatches on primers and tags, respectively. After sequence dereplication using obiuniq, bad-quality sequences (i.e. containing "N"), sequences whose length fell outside the expected size interval (below 45 bp for Bact02, below 68 bp Fung02 and below 36 bp for Euka02) and singletons were filtered out. The obiclean program was run to detect potential PCR or sequencing errors with the $-r$ option set at 0.5: in a PCR reaction, sequences are tagged as "heads" when they are at least twice as abundant as other related sequences differing by one base. Only the sequences tagged as "heads" in at least one PCR were kept.

Taxonomic assignment was conducted using the ecotag program based on a reference database constructed from EMBL (version 136) by running the ecoPCR program (Ficetola et al., 2010). More specifically, ecoPCR carried out an in silico PCR with the primer pair used for the experiment and allowing three mismatches per primer. The obtained reference databases were further curated by keeping only the sequences assigned at least at the family level.

Further data filtering was performed in R version 3.6.1 (R Core Team, 2018) to remove spurious sequences that can bias ecological conclusions drawn from DNA metabarcoding data (Calderón-Sanou et al., 2020). More specifically, we discarded all MOTUs with best identity $<85 \%$ (Fung02, Bact02) or $<80 \%$ (Euka02). These MOTUs were indeed rare ( $1.7 \%$ and $7.3 \%$ of reads for Fung02, Bact02 and Euka02, respectively) and their effect on the NMDS was marginal, as observed in other studies (e.g. Botnen et al. 2018). Furthermore, we removed MOTUS with less than five occurrences in the overall dataset, detected in more than one extraction or PCR negative control (Zinger, Bonin, et al., 2019a), or that were detected in less than two PCR replicates of the same sample, as they often represent false positives (Ficetola et al., 2015).

\section{Statistical analyses}


For all taxonomic groups, we used Generalized Linear Mixed Models (GLMMs) to test if the different treatments lead to differences in the observed MOTU richness. In GLMMs, the number of MOTUs per sample was calculated and used as a dependent effect, the five treatments were used as predictors, and sample identity was used as a random factor. The model was performed with the generalized poisson distribution error using the R package glmmTMB (Brooks et al., 2017), in order to take into account overdispersion (Consul \& Famoye, 1992). If GLMM detected significant differences among treatments, we used treatment contrasts to test if each treatment led to communities significantly different from those unraveled by the "control" condition. Treatment contrasts are standard non-orthogonal contrasts, in which each category (treatment) is compared to a user-defined reference category, and are appropriate to compare multiple treatments against one single control category (in this case, immediate extraction; (Field, Miles, \& Field, 2015). The uncorrected number of MOTUs tends to overestimate the actual taxonomic richness (Calderón-Sanou et al., 2020). Therefore, we repeated this analysis twice: considering all the observed MOTUs, and considering only MOTUs with frequency $\geq 1 \%$ in each sample (hereafter referred to as "common MOTUs").

Subsequently, we used multivariate analyses to assess the variation of bacteria, fungi and eukaryotic communities across habitats and treatments. Before running multivariate analyses, we calculated the proportion of reads of each MOTU in each sample. Relative abundance values were then transformed using the Box-Cox transformation, which simultaneously solves the double-zero problem and improves the multivariate normality of data (Legendre \& Borcard, 2018).

First, we used Nonmetric MultiDimensional Scaling (NMDS) to describe differences in communities among the three habitats, and check whether different treatments yield different interpretations of ecological relationships among samples. NMDS uses an optimization process to find a configuration of points (samples) in a space with a small number of dimensions, and is suitable for metabarcoding analyses that aim to reconstruct variation in community composition as well as possible, without preserving any particular distance measure among objects (Borcard, Gillet, \& Legendre, 2011; Chen \& Ficetola, 2020; Paliy \& Shankar, 2016). Given its robustness and flexibility, NMDS is often used as the first step to characterize 
the similarity of communities in metabarcoding studies (Chen \& Ficetola, 2020; Paliy \& Shankar, 2016). NMDS was run on the Euclidean distance computed on Box-Cox-chord-transformed data (Legendre \& Borcard, 2018), by building 1,000 ordinations.

Second, we used ProcMod, a Procrustes-based analysis (Coissac \& Gonindard-Melodelima, 2019), to measure the multivariate correlations between the communities obtained using the different treatments. ProcMod can be used to measure the shared variation between matrices, and is particularly appropriate to test relationships between datasets obtained through DNA metabarcoding and metagenomics (Coissac \& Gonindard-Melodelima, 2019). Procrustes analyses tend to overfit the data, therefore we used a modified version of Procrustes correlation that is robust to highly-dimensional data and allows a correct estimation of the shared variation between data sets (Coissac \& GonindardMelodelima, 2019). The Procrustes-based correlation tests were performed using the corls function in the $R$ package ProcMod, using 1,000 randomizations to test the mean covariance between random matrices (Coissac \& Gonindard-Melodelima, 2019).

Third, we used redundancy analysis (RDA) to measure the amount of variation among communities that is explained by differences in habitat and treatments (Legendre \& Legendre, 2012; Ter Braak, 1986). With habitat typology and treatment as constraining matrices, we used treatment contrasts to test if each treatment led to communities significantly different from those unraveled by the control treatment. Thus, significant treatment contrasts indicate that results between control and experimental treatments differ in an important way, while non-significant results mean that deviation from ideal conditions is not specifically pronounced. Significance of RDA and treatment contrasts was tested through 10,000 permutations using the vegan package in $\mathrm{R}$ (Borcard et al., 2011; Oksanen et al., 2019).

For bacteria only, RDA detected significant differences between the control and some of the treatments. We thus ran a similarity percentage analysis with the simper $\mathrm{R}$ function (Clarke, 1993) from vegan to identify the taxa contributing to the overall pairwise treatment difference (Geyer et al., 2014). Significance was tested using 50,000 permutations. Given the large number of tests performed, the significance of tests was corrected using the False Discovery Rate (FDR) method with the fdrtool package 
276 (Strimmer, 2008). FDR has greater power than traditional approaches (e.g. Bonferroni correction) when

277 performing multiple comparisons (Benjamini \& Hochberg, 1995). All statistical analyses were performed in 278 the R environment.

279 
A total of $6.3,7.9$ and 25.7 million reads were obtained from the Bact02, Fung02 and Euka02 libraries, respectively. After read assembly, quality filtering, spurious sequence and contaminant removal, 481,411; 2,511,721 and 13,232,441 good-quality sequences remained, consisted of 660 (Bact02), 1,075 (Fung02) and 3,611 (Euka02) unique sequences (i.e. MOTUs).

\section{Differences in MOTU richness among treatments}

Generalized Linear Mixed Models allowed identifying shifts in the richness of observed MOTUs.

When we considered all the detected MOTUs, GLMM detected significant differences in MOTUs richness among treatments for all the markers considered (Bact02: $\chi_{4}=38.9, P<0.001 ;$ Fung02: $\chi_{4}=18.2, P=0.001$; contrasts: Table S1). 
307 Nonmetric MultiDimensional Scaling showed a stress value of 0.13 for Bact02, 0.14 for Fung02 and 0.12 for 308 Euka02. For each of the three markers, the NMDS plots obtained for the five sample preservation treatments were extremely similar, and the ecological differences among the three habitats were clearly identified by all the preservation treatments (Fig. 3).

The multivariate correlation between the communities obtained with the five treatments was always very strong (Procrustes-modified correlation: for all comparisons between "control" and treatments $r \geq 0.84, P<0.0001$; Fig. 4) indicating, for all markers, that most of the variation of retrieved communities was shared across all the treatments. Procrustes correlations were particularly high for Fung02 and Euka02 (all $r \geq 0.9$ ), and between the control and the treatments $6 \mathrm{~h}$ and $4^{\circ} \mathrm{C}$ (all $r \geq 0.93$; Fig. 4).

\section{Differences between the obtained communities}

Redundancy analysis allowed us to measure the amount of variation explained by differences among habitats and by treatments. Overall, $33 \%, 24 \%$, and $33 \%$ of variability was explained by differences in habitat for bacteria, fungi, and eukaryotes, respectively. The community differences among habitats were strongly significant for the three taxonomic groups (permutation test: all $P \leq 0.001$ ). Differences among treatments were much weaker, and explained $9 \%, 2 \%$ and $2 \%$ of variation only for bacteria, fungi and eukaryotes, respectively. Differences between treatments were significant for bacteria (permutation test: $P$ $<0.0001$ ), but not for fungi and eukaryotes (both $P=1$ ).

For bacteria, contrasts did not detect significant differences between control and the $6 \mathrm{~h}$ or $4^{\circ} \mathrm{C}$ treatments. Differences between control and the two silicagel treatments were significant but explained a limited amount of variation (for both treatments, $\approx 3 \%$ of variation explained; $P<0.0001 ;$ Table 1 ). We thus used similarity percentage analysis to identify the MOTUs significantly contributing to these differences. Only one single MOTU showed a significant contribution ( $P=0.03$ after FDR correction) to the differences between control and silicagel treatment; this MOTU (belonging to the Bacteroidetes phylum) showed a 
331 very limited frequency under the silicagel treatment (Fig. S1). After FDR correction, no MOTU showed a

332 significant contribution to the differences between control and the silicagel+6h treatment.

333 


\section{DISCUSSION}

Monitoring soil biodiversity with eDNA metabarcoding over large geographical and taxonomic scales and sometimes in remote places is increasingly important in ecological research. Understanding how preservation conditions affect estimates of taxonomic richness and community composition is essential to ensure sound conclusions. Our study shows that soil metabarcoding results are surprisingly robust to preservation conditions, as we observed limited differences in community structure and diversity estimates when samples were preserved using different strategies. However, some taxonomic groups and diversity components are more sensitive than others to certain preservation conditions. This allowed us to develop guidelines for preservation depending on the aims of monitoring programs and on focal taxa. The aim of this study was comparing realistic approaches to soil preservation against an ideal situation. Immediate extraction was our reference approach, as it avoids both DNA degradation (i.e. potential under-representation of certain taxa) and continued growth of certain taxonomic groups (i.e. potential over-representation of other taxa). Unfortunately, immediate extraction is only possible if sampling occurs nearby facilities, or when a mobile eDNA laboratory is available (e.g. Zinger, Taberlet, et al. 2019b), and logistical constraints often hamper its application in remote areas. We selected preservation conditions among the most achievable, cost-effective and frequent practices to sampling soil for eDNA studies (Dickie et al., 2018), although additional storage methods (e.g. liquid nitrogen, dry ice, RNA later) are available.

\section{Influence of preservation methods on richness estimates}

Preservation methods generated some small but significant differences in MOTUs richness compared to what is observed in the "control", with some contrasting effects across taxa. When considering all the MOTUs, none of the preservation conditions yielded estimates of alpha-diversity identical to the "control". For instance, just six hours at room temperature caused a significant decrease of MOTUs richness in fungi. It has been shown that estimates of alpha-diversity using metabarcoding are extremely sensitive to methodological choices (Calderón-Sanou et al., 2020). Our study underlines that even preservation for a 
very short time can affect the detection of rare MOTUs and highlights the sensitivity of fungi to preservation at room temperature (Delavaux, Bever, Karppinen, \& Bainard, 2020). MOTUs richness of all the taxa was also affected by preservation at $4^{\circ} \mathrm{C}$, which caused a slight increase of MOTUs richness for bacteria and eukaryotes, and a slight decrease for fungi. The effect of temperature and time storage in fungal and bacterial growth has already been proven (see e.g. Orchard et al., 2017; Pettersson \& Bååth, 2003). Despite this, in addition to temperature, we can expect that other parameters such as initial soil moisture and pH influence bacterial growth (Bååth \& Arnebrant, 1994; Drenovsky, Vo, Graham, \& Scow, 2004; Fernández-Calviño \& Bååth, 2010; Kaiser et al., 2016) with a combined effect. Finally, drought affects the richness of microbial communities in soil ecosystems with differential effects across taxa depending on their ecology (Evans, Wallenstein, \& Burke, 2014; Meisner, Jacquiod, Snoek, Ten Hooven, \& van der Putten, 2018; Ochoa-Hueso et al., 2018), and three weeks of preservation with silica gel generally reduced the observed MOTUs richness in bacteria and fungi, while it increased the richness of eukaryotes. However, our study also shows that specific caution is mostly necessary when rare MOTUs are of interest. The exclusion of rare and uncommon MOTUs strongly reduced differences between optimal conditions and different preservation. The remaining effects were much weaker for bacteria and fungi, while disappeared for eukaryotes (Fig. 2), and can be due to the heterogeneous growth across taxonomic groups, or to differential DNA degradation under different preservation conditions. This suggests that the effect of preservation approach on taxonomic richness mostly occurs on rare species, as already suggested for microbial communities (Meisner et al. 2018). Several authors have shown that eDNA metabarcoding does not represent the best tool for the detection of rare MOTUs, as some rare MOTUs remain undetected, while many sequences detected at rare frequency are artifacts (Brown et al., 2015). Estimates of $\alpha$-diversity should therefore always be taken with caution, and indices that underweight rare MOTUs (e.g. Shannon or Simpson diversity) can provide more robust estimates (Brown et al., 2015; Calderón-Sanou et al., 2020; Os B' Alint et al., 2016).

\section{Differences in community structure}


If the study interest is in community structure and not in richness estimates, then preservation choices

387 become even less important. In fact, the similarity of communities obtained through the different 388 preservation conditions is surprisingly high (see Procrustes correlation coefficients; Fig. 4); the amount of variation explained by preservation conditions was much lower than the observed differences among habitats (see redundancy analysis), and multivariate analyses consistently allowed to detect community differences among habitats (Fig. 3). In other words, metabarcoding is able to identify the ecological differences among sites, independently of the preservation approach. Even though metabarcoding analyses are sensitive to methodological choices, estimates of relationships between diversity and the environment are often robust (Calderón-Sanou et al., 2020; Ji et al., 2013), and this is a very good news if we want to apply these approaches to broad-scale monitoring programs, aiming at assessing the effects of environmental changes. Bacteria were the only taxon for which we detected significant differences between the "control" and the preservation conditions, with $\approx 3 \%$ of variability explained by differences between the "control" and the desiccation treatments. The observed differences most likely refer to some taxa that are affected by the dry conditions and could lead to an overrepresentation of some taxa that are more resistant under these conditions. We expect some taxa to better survive and grow in dry treatments with respect to others, and this would increase make their DNA more available in the samples. Differences between the desiccation treatments and the control were small, and only one out of 660 MOTUs showed a significant variation in abundance with the control. This MOTU (belonging to the Bacteroidetes phylum of bacteria, see Supporting Information) was generally abundant in the control and preservation conditions 2 and 3 (average frequency of reads around 10\%) while it drastically decreased under preservation conditions 4 and 5 (Fig. S1). This agrees with studies showing that different genera belonging to this phylum respond differently to drought (Meisner et al. 2018). In fact, the Bacteroidetes Flavobacterium shows an increase in abundance over dried-treatments, even though differences after three weeks were not significant (see Fig. S2). 
importance of selecting the preservation method before starting a monitoring program and using it consistently through the whole monitoring, to avoid confusion between the effects of methods and of environmental changes.

\section{Conclusions: guidelines for optimizing preservation conditions}

417 Standardized protocols are essential for repeatable and reliable biodiversity monitoring, and our results

418 allow to propose guidelines to improve and standardize the preservation of soil samples for eDNA 419 metabarcoding analyses (Fig. 5):

420 1) If sampling occurs close to lab facilities, or a mobile lab is available, extracting DNA as soon as possible 421 is the best approach. Storing samples a few hours at room temperature does not have major impact on the outcome of analyses, especially if the focus is not on rare MOTUs;

2) If lab facilities are available after a short-time transportation, storing samples in the fridge $\left(0-4^{\circ} \mathrm{C}\right)$ for a few days is a safe approach as it does not have a significant impact on community composition, and only moderately affects MOTUs richness. However, this approach can be problematic if the aim is to estimate MOTUs richness, and particularly the occurrence of rare MOTUs. The feasibility of this

3) If monitoring in remote areas, sample desiccation (e.g. using silica gel) and long-term preservation at room temperature is a reasonable approach, and it is particularly convenient when working with a large volume of samples. This approach preserves ecological signal, but can affect the detection of some taxa, particularly among the rarest ones. Therefore, this approach is suboptimal for monitoring programs aiming at detecting rare MOTUs.

An effective application of eDNA metabarcoding to biodiversity monitoring is complex, and protocols of sample preservation are key methodological choices that have to be taken into account when designing a metabarcoding-based monitoring. When working in difficult and remote environments researchers are 
faced with the trade-offs between a faithful representation of biodiversity, and multiple logistic constraints

438 in the field. Accurate a-priori planning is often the basis of successful monitoring programs and our

439 guidelines can help researchers and practitioners to identify the best approach to sample preservation,

depending on the studied taxa and research goals.

\section{ACKNOWLEDGMENTS}

We thank P. Taberlet for constructive discussions. This study is funded by the European Research Council under the European Community's Horizon 2020 Programme, Grant Agreement no. 772284

(IceCommunities). WT, TM and LG also thank the rom the French Agence Nationale de la Recherche (ANR) through the GlobNets (ANR-16-CE02-0009) project and from 'Investissement d'Avenir' grant managed by the ANR (Montane: OSUG@2020: ANR-10-LAB-56).

\section{REFERENCES}

Guerrieri, Alessia et al. (2020), Effects of soil preservation for biodiversity monitoring using environmental DNA, Dryad, Dataset, https://doi.org/10.5061/dryad.zkh189382

Bååth, E., \& Arnebrant, K. (1994). Growth rate and response of bacterial communities to $\mathrm{pH}$ in limed and ash treated forest soils. Soil Biology and Biochemistry, 26(8), 995-1001. doi: 10.1016/00380717(94)90114-7

Benjamini, Y., \& Hochberg, Y. (1995). Controlling the False Discovery Rate: A Practical and Powerful Approach to Multiple Testing. Journal of the Royal Statistical Society: Series B (Methodological), 57(1), 289-300. doi: 10.1111/j.2517-6161.1995.tb02031.x

Borcard, D., Gillet, F., \& Legendre, P. (2011). Numerical Ecology with R. In Numerical Ecology with R. doi: 10.1007/978-1-4419-7976-6

Boyer, F., Mercier, C., Bonin, A., Le Bras, Y., Taberlet, P., \& Coissac, E. (2016). obitools: A unix-inspired software package for DNA metabarcoding. Molecular Ecology Resources, 16(1), 176-182. doi: 10.1111/1755-0998.12428

Brooks, M. E., Kristensen, K., van Benthem, K. J., Magnusson, A., Berg, C. W., Nielsen, A., ... Bolker, B. M. (2017). glmmTMB balances speed and flexibility among packages for zero-inflated generalized linear mixed modeling. $R$ Journal, 9(2), 378-400. doi: 10.32614/rj-2017-066

Brown, S. P., Veach, A. M., Rigdon-Huss, A. R., Grond, K., Lickteig, S. K., Lothamer, K., ... Jumpponen, A. (2015). Scraping the bottom of the barrel: Are rare high throughput sequences artifacts? Fungal Ecology, 13, 221-225. doi: 10.1016/j.funeco.2014.08.006

Calderón-Sanou, I., Münkemüller, T., Boyer, F., Zinger, L., \& Thuiller, W. (2020). From environmental DNA sequences to ecological conclusions: How strong is the influence of methodological choices? Journal of Biogeography, 47(1), 193-206. doi: 10.1111/jbi.13681

Cantera, I., Cilleros, K., Valentini, A., Cerdan, A., Dejean, T., Iribar, A., ... Brosse, S. (2019). Optimizing environmental DNA sampling effort for fish inventories in tropical streams and rivers. Scientific Reports, 9(1), 1-11. doi: 10.1038/s41598-019-39399-5

Cardona, S., Eck, A., Cassellas, M., Gallart, M., Alastrue, C., Dore, J., ... Manichanh, C. (2012). Storage conditions of intestinal microbiota matter in metagenomic analysis. BMC Microbiology, 12. doi: 10.1186/1471-2180-12-158

Chase, M. W., \& Hills, H. H. (1991). Silica gel: An ideal material for field preservation of leaf samples for DNA studies. TAXON, 4O(2), 215-220. doi: 10.2307/1222975

Chen, W., \& Ficetola, G. F. (2020). Numerical methods for sedimentary-ancient-DNA-based study on past biodiversity and ecosystem functioning. Environmental DNA, edn3.79. doi: 10.1002/edn3.79 
CLARKE, K. R. (1993). Non-parametric multivariate analyses of changes in community structure. Austral Ecology, 18(1), 117-143. doi: 10.1111/j.1442-9993.1993.tb00438.x

Coissac, E. (2012). OligoTag: A program for designing sets of tags for next-generation sequencing of multiplexed samples. Methods in Molecular Biology, 888, 13-31. doi: 10.1007/978-1-61779-870-2_2

Coissac, E., \& Gonindard-Melodelima, C. (2019). Assessing the shared variation among high-dimensional data matrices: a modified version of the Procrustean correlation coefficient. BioRxiv Bioinformatics, 116. doi: $10.1101 / 842070$

Consul, P. C., \& Famoye, F. (1992). Generalized poisson regression model. Communications in Statistics Theory and Methods, 21(1), 89-109. doi: 10.1080/03610929208830766

De Vargas, C., Audic, S., Henry, N., Decelle, J., Mahé, F., Logares, R., ... Velayoudon, D. (2015). Eukaryotic plankton diversity in the sunlit ocean. Science, 348(6237). doi: 10.1126/science.1261605

Delavaux, C. S., Bever, J. D., Karppinen, E. M., \& Bainard, L. D. (2020). Keeping it cool: Soil sample cold pack storage and DNA shipment up to 1 month does not impact metabarcoding results. Ecology and Evolution, (November 2019), 1-13. doi: 10.1002/ece3.6219

Delgado-Baquerizo, M., Oliverio, A. M., Brewer, T. E., Benavent-González, A., Eldridge, D. J., Bardgett, R. D., ... Fierer, N. (2018). A global atlas of the dominant bacteria found in soil. Science, 359(6373), 320-325. doi: 10.1126/science.aap9516

Dickie, I. A., Boyer, S., Buckley, H. L., Duncan, R. P., Gardner, P. P., Hogg, I. D., ... Weaver, L. (2018). Towards robust and repeatable sampling methods in eDNA-based studies. Molecular Ecology Resources, 18(5), 940-952. doi: 10.1111/1755-0998.12907

Drenovsky, R. E., Vo, D., Graham, K. J., \& Scow, K. M. (2004). Soil water content and organic carbon availability are major determinants of soil microbial community composition. Microbial Ecology, 48(3), 424-430. doi: 10.1007/s00248-003-1063-2

Epp, L. S., Boessenkool, S., Bellemain, E. P., Haile, J., Esposito, A., Riaz, T., ... Brochmann, C. (2012). New environmental metabarcodes for analysing soil DNA: Potential for studying past and present ecosystems. Molecular Ecology, 21(8), 1821-1833. doi: 10.1111/j.1365-294X.2012.05537.x

Evans, S. E., Wallenstein, M. D., \& Burke, I. C. (2014). Is bacterial moisture niche a good predictor of shifts in community composition under long-term drought. Ecology, 95(1), 110-122. doi: 10.1890/13-0500.1

Fernández-Calviño, D., \& Bååth, E. (2010). Growth response of the bacterial community to pH in soils differing in pH. FEMS Microbiology Ecology, 73(1), 149-156. doi: 10.1111/j.1574-6941.2010.00873.x

Ficetola, G. F., Coissac, E., Zundel, S., Riaz, T., Shehzad, W., Bessière, J., ... Pompanon, F. (2010). An In silico approach for the evaluation of DNA barcodes. BMC Genomics, 11(1), 434. doi: 10.1186/1471-2164-11434

Ficetola, G. F., Pansu, J., Bonin, A., Coissac, E., Giguet-Covex, C., De Barba, M., ... Taberlet, P. (2015). Replication levels, false presences and the estimation of the presence/absence from eDNA metabarcoding data. Molecular Ecology Resources. doi: 10.1111/1755-0998.12338

Field, A. P., Miles, J. 1968-, \& Field, Z. (2012). Discovering statistics Using r. London ; Thousand Oaks, Calif. : Sage,

Geyer, K. M., Altrichter, A. E., Takacs-Vesbach, C. D., Van Horn, D. J., Gooseff, M. N., \& Barrett, J. E. (2014). Bacterial community composition of divergent soil habitats in a polar desert. FEMS Microbiology Ecology, 89(2), 490-494. doi: 10.1111/1574-6941.12306

Giovannoni, S. J., Britschgi, T. B., Moyer, C. L., \& Field, K. G. (1990). Genetic diversity in Sargasso Sea bacterioplankton. Nature, 345(6270), 60-63. doi: 10.1038/345060a0

Guardiola, M., Uriz, M. J., Taberlet, P., Coissac, E., Wangensteen, O. S., \& Turon, X. (2015). Deep-Sea, DeepSequencing: Metabarcoding Extracellular DNA from Sediments of Marine Canyons. PLOS ONE, 10(10), e0139633. doi: 10.1371/journal.pone.0139633

Guo, Y., Yang, G. Q., Chen, Y., Li, D., \& Guo, Z. (2018). A comparison of different methods for preserving plant molecular materials and the effect of degraded DNA on ddRAD sequencing. Plant Diversity, 40(3), 106-116. doi: 10.1016/j.pld.2018.04.001

Hoffmann, C., Schubert, G., \& Calvignac-Spencer, S. (2016, February 1). Aquatic biodiversity assessment for the lazy. Molecular Ecology, Vol. 25, pp. 846-848. doi: 10.1111/mec.13535

Huerlimann, R., Cooper, M. K., Edmunds, R. C., Villacorta-Rath, C., Le Port, A., Robson, H. L. A., ... Jerry, D. R. 
(2020). Enhancing tropical conservation and ecology research with aquatic environmental DNA methods: an introduction for non-environmental DNA specialists. Animal Conservation. doi: 10.1111/acv.12583

Ji, Y., Ashton, L., Pedley, S. M., Edwards, D. P., Tang, Y., Nakamura, A., ... Yu, D. W. (2013). Reliable, verifiable and efficient monitoring of biodiversity via metabarcoding. Ecology Letters, 16(10), 12451257. doi: $10.1111 /$ ele.12162

Kaiser, K., Wemheuer, B., Korolkow, V., Wemheuer, F., Nacke, H., Schöning, I., ... Daniel, R. (2016). Driving forces of soil bacterial community structure, diversity, and function in temperate grasslands and forests. Scientific Reports, 6(August), 1-12. doi: 10.1038/srep33696

Kumar, G., Eble, J. E., \& Gaither, M. R. (2020). A practical guide to sample preservation and pre-PCR processing of aquatic environmental DNA. Molecular Ecology Resources, 20(1), 29-39. doi: 10.1111/1755-0998.13107

Lauber, C. L., Zhou, N., Gordon, J. I., Knight, R., \& Fierer, N. (2010). Effect of storage conditions on the assessment of bacterial community structure in soil and human-associated samples. FEMS Microbiology Letters, 307(1), 80-86. doi: 10.1111/j.1574-6968.2010.01965.x

Legendre, P., \& Borcard, D. (2018). Box-Cox-chord transformations for community composition data prior to beta diversity analysis. Ecography, 41(11), 1820-1824. doi: 10.1111/ecog.03498

Legendre, P., \& Legendre, L. (2012). Numerical Ecology Ch 6 - Multidimensional qualitative data. Developments in Environmental Modelling, 24, 337-424. doi: 10.1016/B978-0-444-53868-0.50008-3

Majaneva, M., Diserud, O. H., Eagle, S. H. C., Boström, E., Hajibabaei, M., \& Ekrem, T. (2018). Environmental DNA filtration techniques affect recovered biodiversity. Scientific Reports, 8(1), 4682. doi: 10.1038/s41598-018-23052-8

Meisner, A., Jacquiod, S., Snoek, B. L., Ten Hooven, F. C., \& van der Putten, W. H. (2018). Drought legacy effects on the composition of soil fungal and prokaryote communities. Frontiers in Microbiology, 9(MAR), 294. doi: 10.3389/fmicb.2018.00294

Nichols, R. V., Vollmers, C., Newsom, L. A., Wang, Y., Heintzman, P. D., Leighton, M., ... Shapiro, B. (2018). Minimizing polymerase biases in metabarcoding. Molecular Ecology Resources, 18(5), 927-939. doi: 10.1111/1755-0998.12895

Ochoa-Hueso, R., Collins, S. L., Delgado-Baquerizo, M., Hamonts, K., Pockman, W. T., Sinsabaugh, R. L., ... Power, S. A. (2018). Drought consistently alters the composition of soil fungal and bacterial communities in grasslands from two continents. Global Change Biology, 24(7), 2818-2827. doi: 10.1111/gcb.14113

Oksanen, J., Blanchet, F. G., Friendly, M., Kindt, R., Legendre, P., Mcglinn, D., ... Maintainer, H. W. (2019). Package "vegan" Title Community Ecology Package Version 2.5-6.

Orchard, S., Standish, R. J., Nicol, D., Dickie, I. A., \& Ryan, M. H. (2017). Sample storage conditions alter colonisation structures of arbuscular mycorrhizal fungi and, particularly, fine root endophyte. Plant and Soil, 412(1-2), 35-42. doi: 10.1007/s11104-016-2867-4

Os B ' Alint, M., Bahram, M., Murat Eren, A., Faust, K., Fuhrman, J. A., Bj”, B., ... Tedersoo, L. (2016). Millions of reads, thousands of taxa: microbial community structure and associations analyzed via marker genes. FEMS Microbiology Reviews, 017, 686-700. doi: 10.1093/femsre/fuw017

Paliy, O., \& Shankar, V. (2016, March 1). Application of multivariate statistical techniques in microbial ecology. Molecular Ecology, Vol. 25, pp. 1032-1057. doi: 10.1111/mec.13536

Pettersson, M., \& Bååth, E. (2003). Temperature-dependent changes in the soil bacterial community in limed and unlimed soil. FEMS Microbiology Ecology, 45(1), 13-21. doi: 10.1016/S01686496(03)00106-5

Spens, J., Evans, A. R., Halfmaerten, D., Knudsen, S. W., Sengupta, M. E., Mak, S. S. T., ... Hellström, M. (2017). Comparison of capture and storage methods for aqueous macrobial eDNA using an optimized extraction protocol: advantage of enclosed filter. Methods in Ecology and Evolution, 8(5), 635-645. doi: 10.1111/2041-210X.12683

Strimmer, K. (2008). fdrtool: a versatile R package for estimating local and tail area-based false discovery rates. Bioinformatics (Oxford, England), 24(12), 1461-1462. doi: 10.1093/bioinformatics/btn209

Taberlet, P., Bonin, A., Zinger, L., \& Coissac, É. (2018). Environmental DNA: For Biodiversity Research and 
Monitoring. In Environmental DNA: For Biodiversity Research and Monitoring. doi: 10.1093/oso/9780198767220.001.0001

TABERLET, P., COISSAC, E., POMPANON, F., BROCHMANN, C., \& WILLERSLEV, E. (2012). Towards nextgeneration biodiversity assessment using DNA metabarcoding. Molecular Ecology, 21(8), 2045-2050. doi: 10.1111/j.1365-294X.2012.05470.x

Ter Braak, C. J. F. (1986). Canonical correspondence analysis: a new eigenvector technique for multivariate direct gradient analysis. Ecology, 67(5), 1167-1179. doi: 10.2307/1938672

Ward, D. M., Weller, R., \& Bateson, M. M. (1990). 16S rRNA sequences reveal numerous uncultured microorganisms in a natural community. Nature, 345(6270), 63-65. doi: 10.1038/345063a0

Zinger, L., Bonin, A., Alsos, I. G., Bálint, M., Bik, H., Boyer, F., ... Taberlet, P. (2019, April 1). DNA metabarcoding-Need for robust experimental designs to draw sound ecological conclusions. Molecular Ecology, Vol. 28, pp. 1857-1862. doi: 10.1111/mec.15060

Zinger, L., Taberlet, P., Schimann, H., Bonin, A., Boyer, F., De Barba, M., ... Chave, J. (2019). Body size determines soil community assembly in a tropical forest. Molecular Ecology, 28(3), 528-543. doi: 10.1111/mec.14919

\section{DATA ACCESSIBILITY}

Raw sequences as well as filtered data are available in the DRYAD Digital Repository

(https://doi.org/10.5061/dryad.zkh189382)

\section{AUTHOR CONTRIBUTIONS}

TM, GFF, LG, AB, WT and AG designed the experiment. LG, TM and AG conducted the field work. AG conducted all molecular analyses and performed the bioinformatic treatment of sequences with the help of $A B$. AG, $A B$ and GFF ran statistical analyses. AG, GFF and $A B$ drafted the manuscript. All the authors contributed substantially to the revision process, and accepted the final version.

\section{ORCID}

Alessia Guerrieri: https://orcid.org/0000-0002-1519-3517

Aurélie Bonin: https://orcid.org/0000-0001-7800-8609

Tamara Münkemüller: https://orcid.org/0000-0001-9743-1322

Ludovic Gielly: https://orcid.org/0000-0001-5164-6512

Wilfried Thuiller: https://orcid.org/0000-0002-5388-5274

Gentile Francesco Ficetola: https://orcid.org/0000-0003-3414-5155 
621 Table 1. Treatment contrasts assessing differences between the control (immediate extraction) and four 622 approaches to soil conservation before eDNA extraction. Each conservation treatment was compared 623 against the control in order to determine the percentage of explained variability.

\begin{tabular}{|c|c|c|c|c|c|c|}
\hline & \multicolumn{2}{|c|}{ Bact02 } & \multicolumn{2}{|c|}{ Fung02 } & \multicolumn{2}{|c|}{ Euka02 } \\
\hline & $\begin{array}{l}\text { Explained } \\
\text { variability (\%) }\end{array}$ & $P$ & $\begin{array}{l}\text { Explained } \\
\text { variability (\%) }\end{array}$ & $P$ & $\begin{array}{l}\text { Explained } \\
\text { variability (\%) }\end{array}$ & $P$ \\
\hline $\begin{array}{l}\text { Treatment 2: room temperature, extraction } \\
\text { after } 6 \mathrm{~h} \text {; }\end{array}$ & 0.58 & 0.956 & 0.53 & 1 & 0.44 & 0.993 \\
\hline Treatment 3: $4^{\circ} \mathrm{C}$, extraction after 3 days; & 0.81 & 0.563 & 0.58 & 0.976 & 0.54 & 0.949 \\
\hline $\begin{array}{l}\text { Treatment 4: silica gel immediately inserted, } \\
\text { extraction after } 21 \text { days; }\end{array}$ & 3.14 & $<0.001$ & 1.03 & 0.518 & 0.63 & 0.876 \\
\hline $\begin{array}{l}\text { Treatment 5: silica gel inserted after } 6 \mathrm{~h} \text {, } \\
\text { extraction after } 21 \text { days; }\end{array}$ & 3.16 & $<0.001$ & 0.73 & 0.880 & 0.75 & 0.725 \\
\hline
\end{tabular}

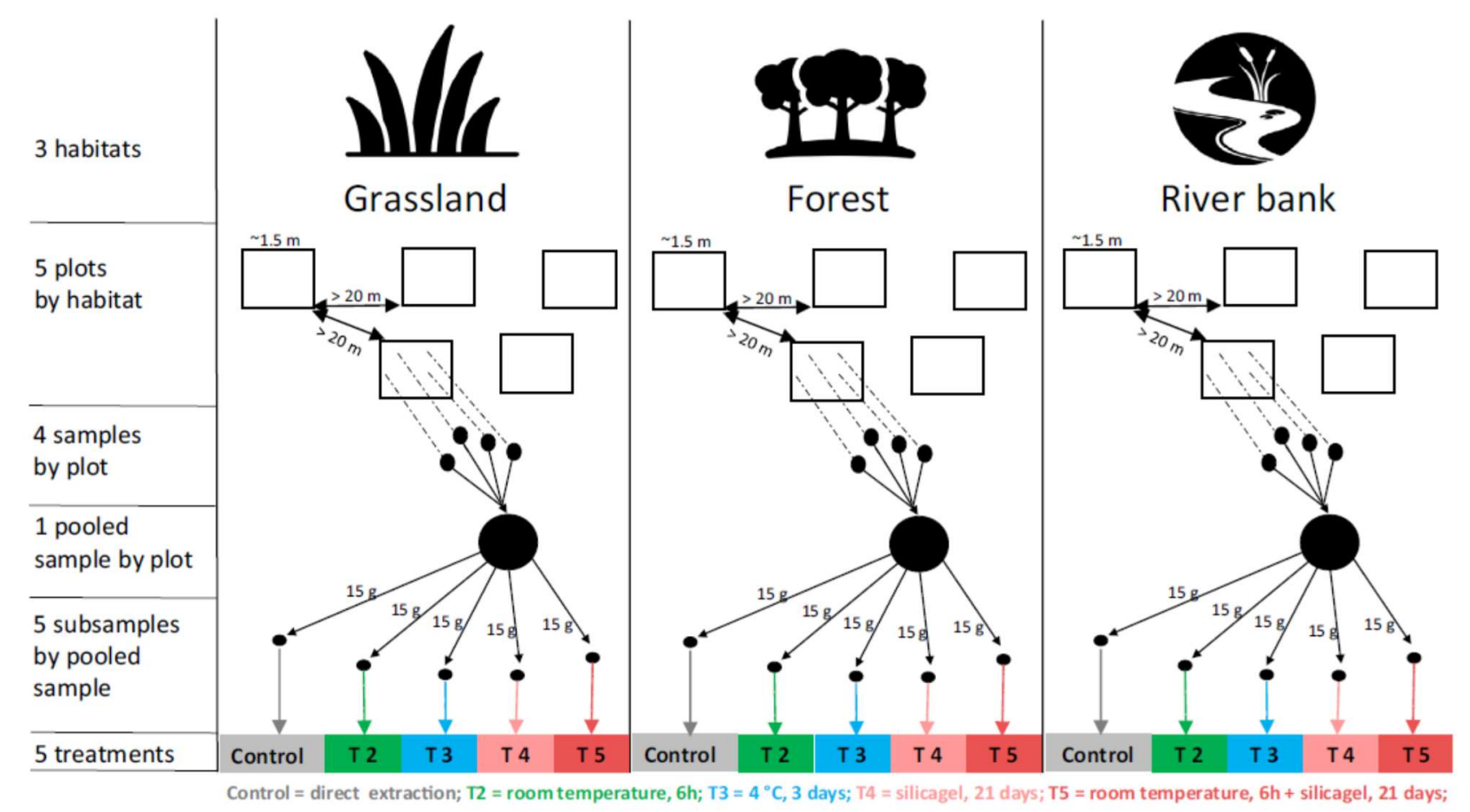


625 Fig. 1. Experimental sampling design.

626 
With rare MOTUs
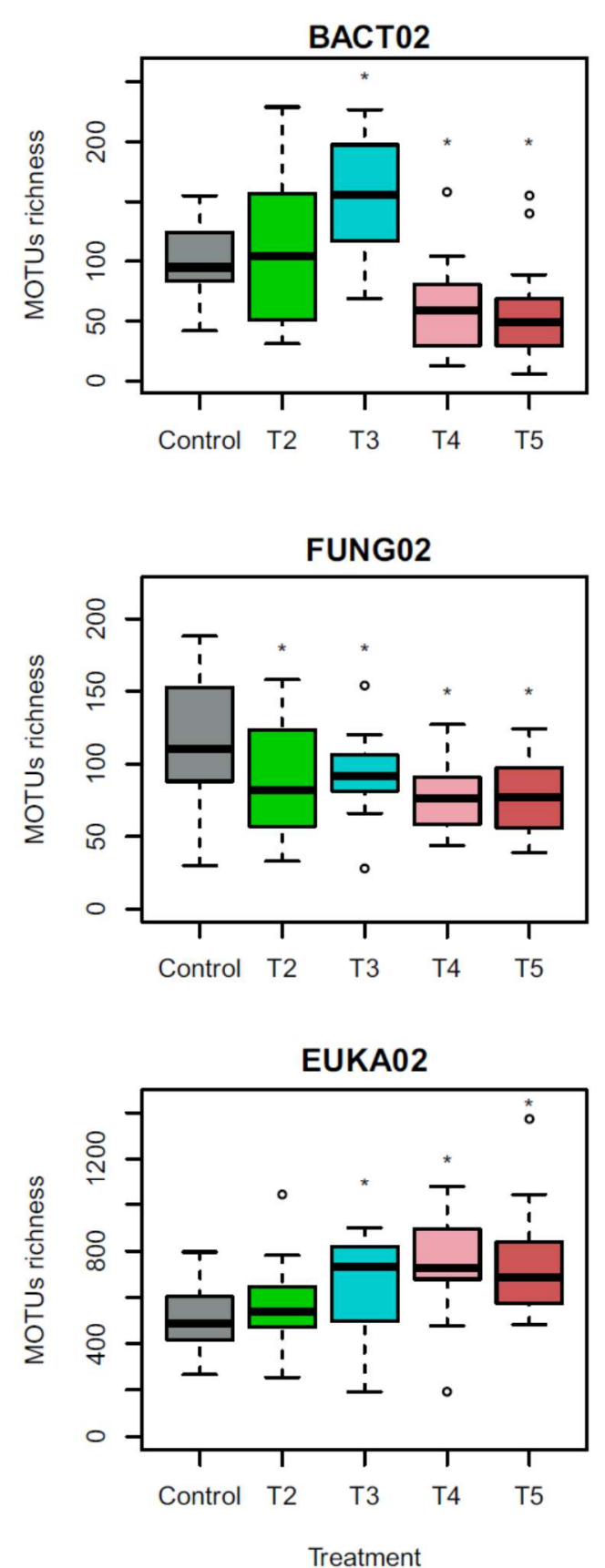

Without rare MOTUs

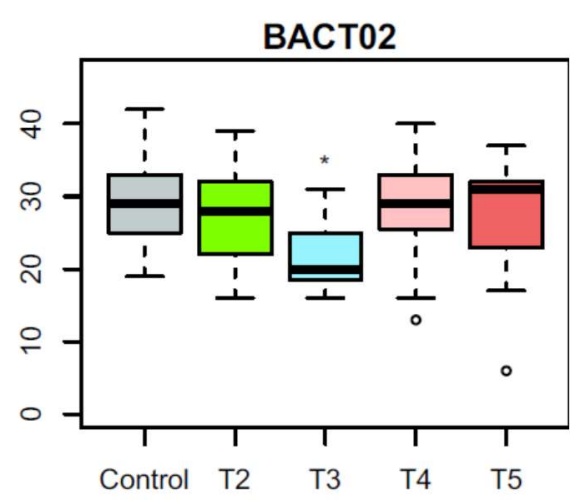

FUNG02

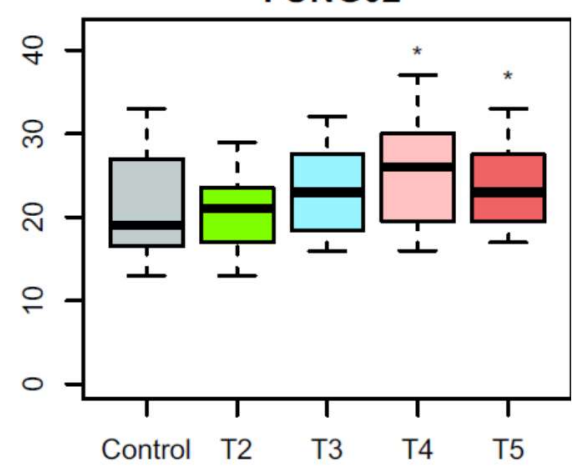

EUKA02

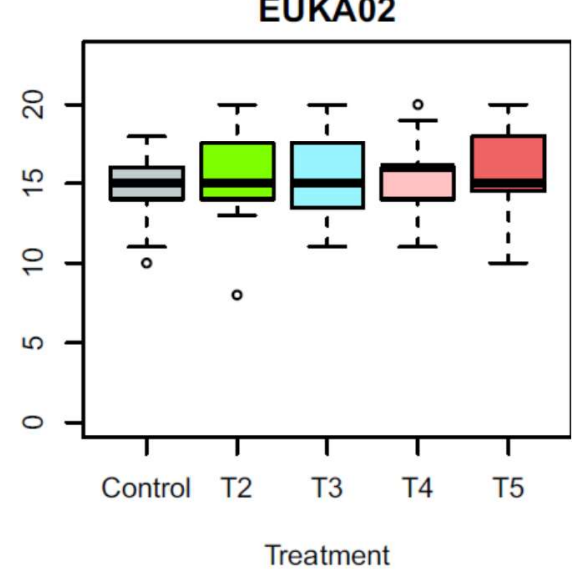

Fig. 2. MOTUs richness across the different treatments (control: immediate extraction; T2: extraction after $6 \mathrm{~h}$ at room temperature; T3: extraction after three days at $4^{\circ} \mathrm{C}$; T4: immediate preservation in tubes with silica gel, extraction after 21 days; T5: preservation in tubes with silica gel after $6 \mathrm{~h}$ at room temperature, extraction after 21 days) before (left) and after (right) removing MOTUs with frequency $<1 \%$ in each sample. 

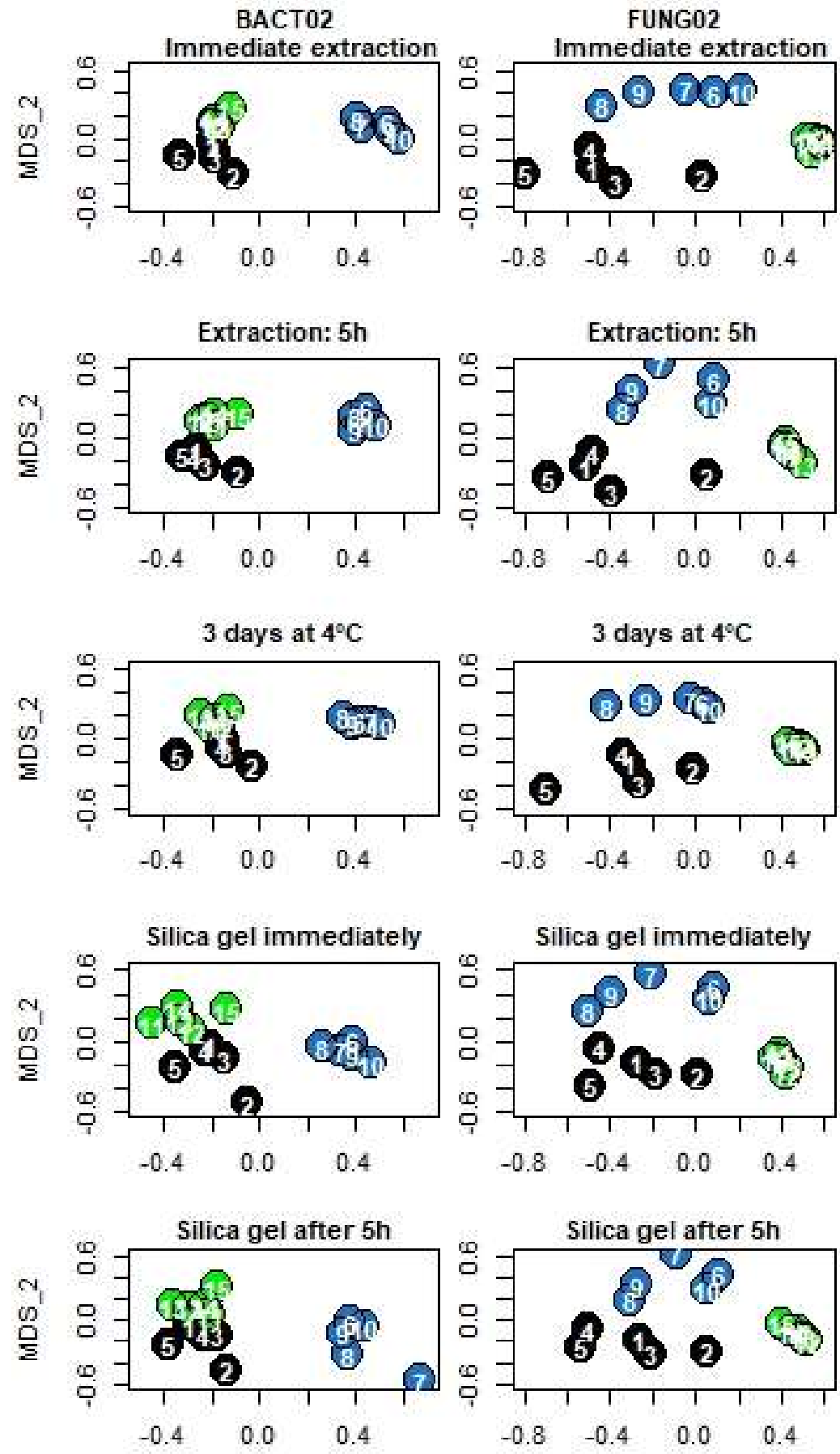

634

635

636

637

638

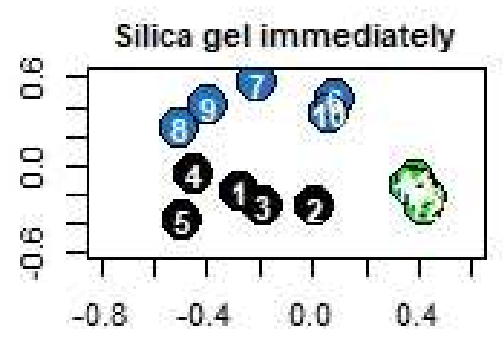

FUNG02

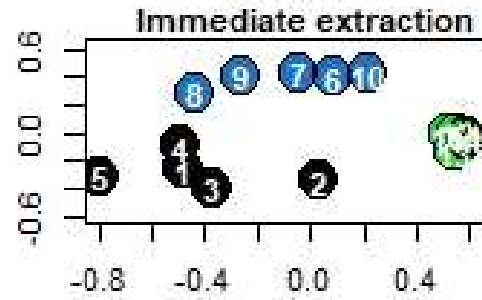

Extraction: $5 \mathrm{~h}$
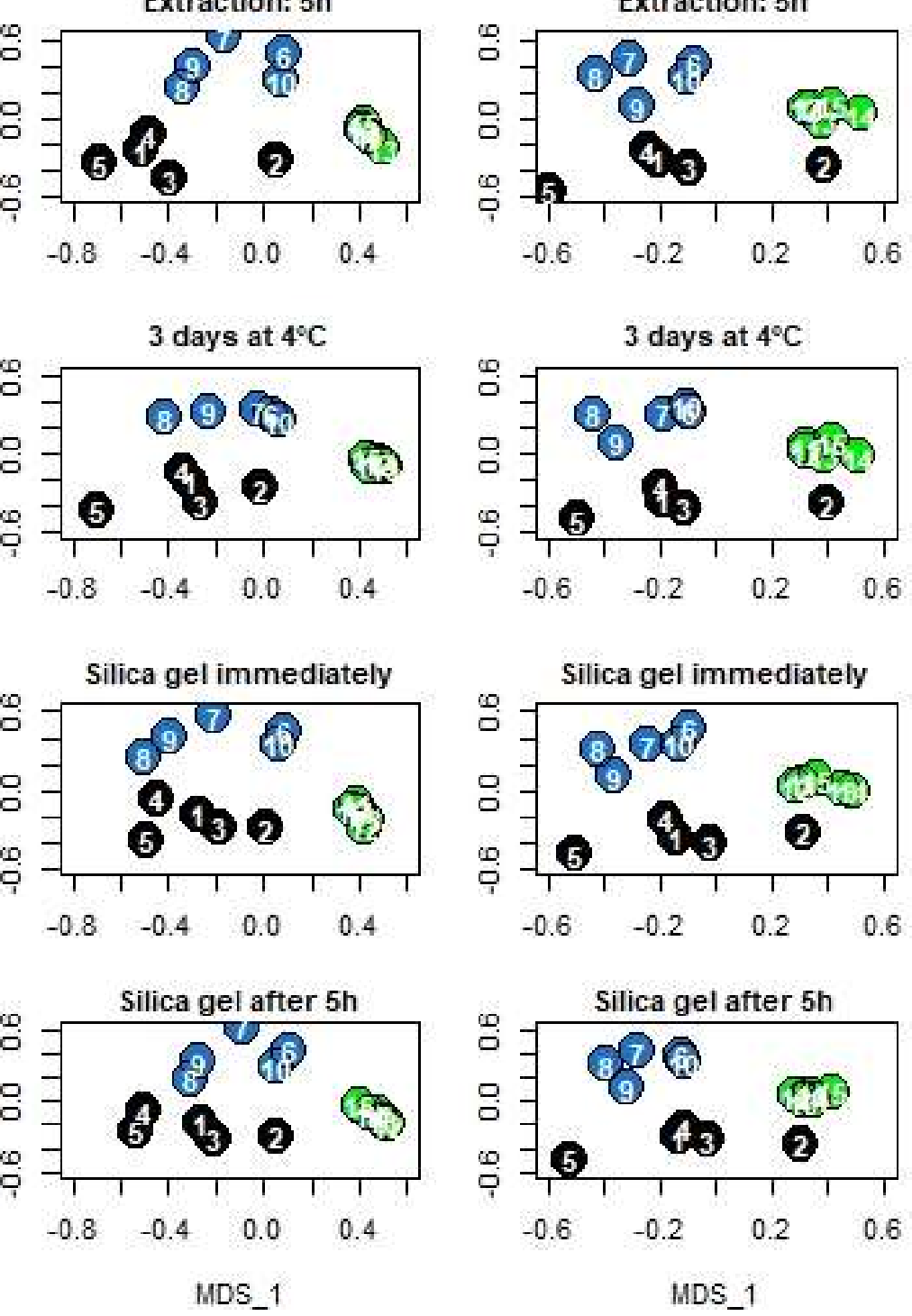

Fig. 3. Plots of non-metric dimensional scaling showing dissimilarities of communities among the three habitats: broadleaved forest (black); grassland (green); vegetated riverbank (blue). Each plot shows the results of metabarcoding analysis based on soil samples subjected to five different treatments. 
ВАСТ02

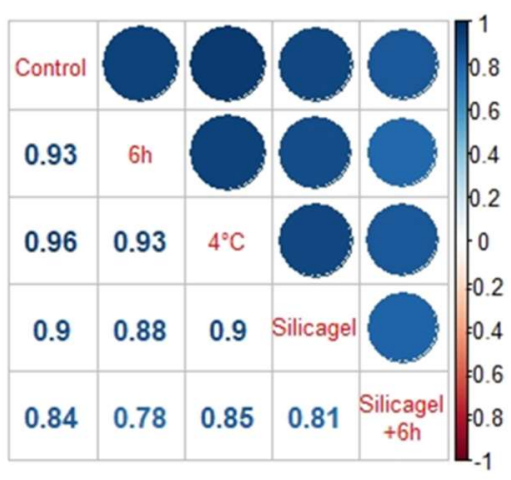

FUNG02

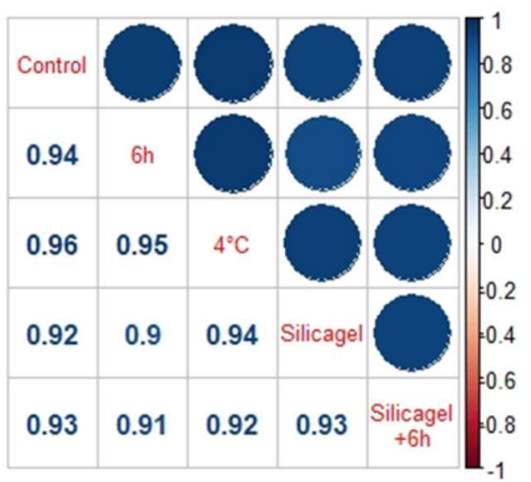

EUKA02

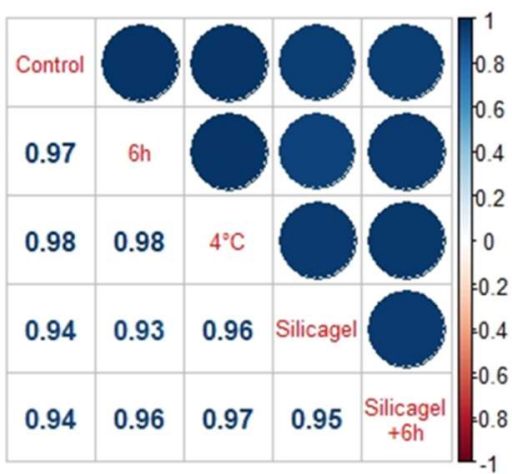

639

640

641

642

643

644

645

646

Fig. 4. Procrustes correlation between communities obtained from metabarcoding analyses based on soil samples across environmental conditions subjected to five sample treatments (control: immediate extraction; $\mathrm{RT}+6 \mathrm{~h}$ : extraction after $6 \mathrm{~h}$ at room temperature; $4^{\circ} \mathrm{C}$ : extraction after three days at $4^{\circ} \mathrm{C}$; silicagel: immediate preservation in tubes with silica gel, extraction after 21 days; silicagel+6h: preservation in tubes with silica gel after $6 \mathrm{~h}$ at room temperature, extraction after 21 days. All correlation coefficients are highly significant (all $\mathrm{P}<0.0001$ ).

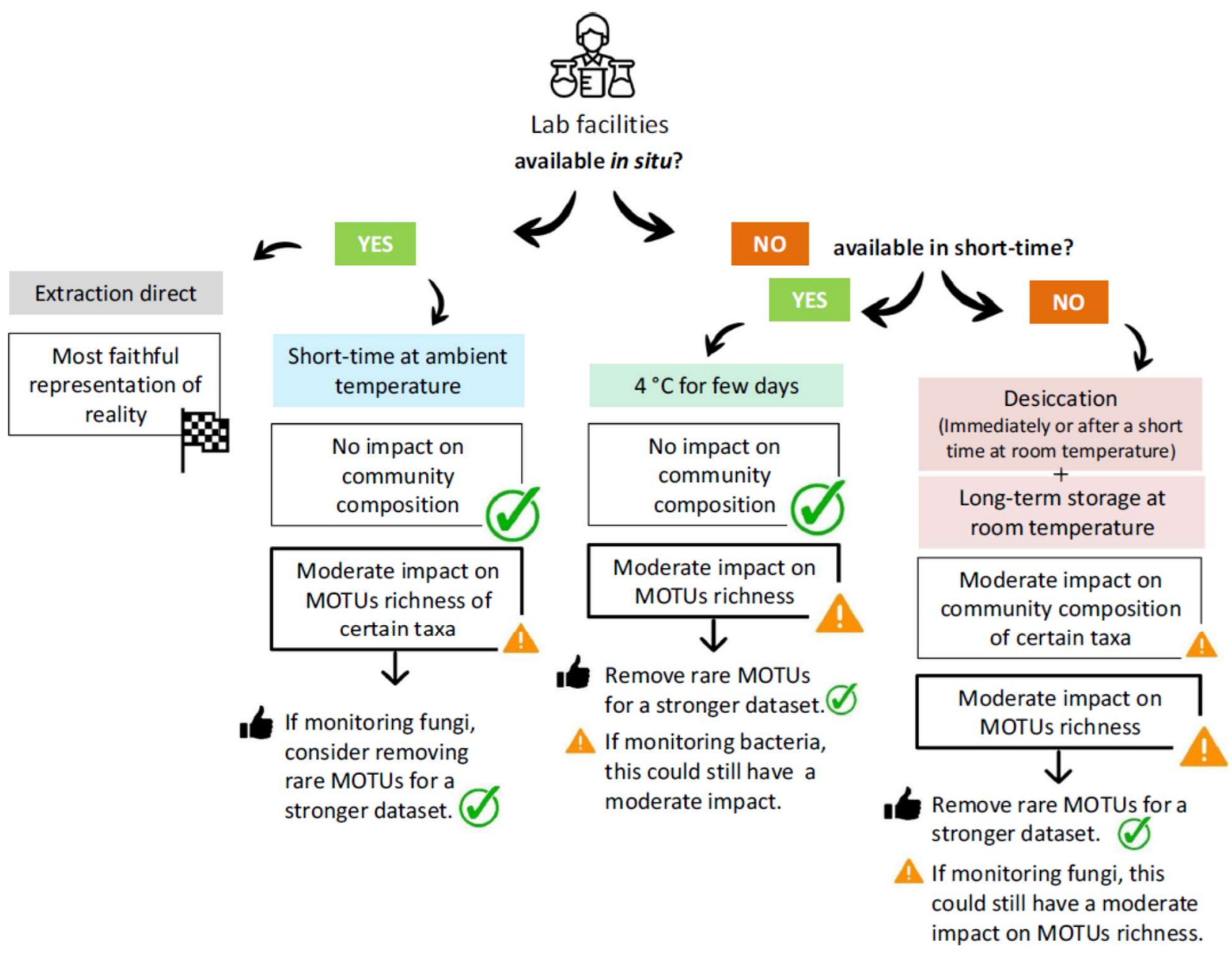

Fig. 5. Guidelines for improving monitoring strategies with eDNA from soil. 